\title{
The Coexistence of Multiple Myeloma-associated Amyloid Light-chain Amyloidosis and Fabry Disease in a Hemodialysis Patient
}

\author{
Kensei Taguchi ${ }^{1}$, Atsuo Moriyama ${ }^{2}$, Goh Kodama $^{1}$, Yosuke Nakayama ${ }^{1}$ and Kei Fukami ${ }^{1}$
}

\begin{abstract}
Fabry disease (FD) is an inherited lysosomal disorder caused by an X-linked $\alpha$-galactosidase A deficiency. We report the case of a 50-year-old male FD patient on hemodialysis who presented with macroglossiarelated speaking difficulty and gastrointestinal symptoms. An endoscopic analysis revealed multiple gastric ulcers, and a histological examination led to a diagnosis of amyloid light-chain amyloidosis. Serum free lightchain and bone marrow analyses detected multiple myeloma (MM). Treatment with bortezomib and dexamethasone significantly improved the patient's symptoms. This is the first case to demonstrate a potential pathogenic relationship between FD and MM. The similar gastrointestinal manifestations might have contributed to the diagnostic difficulty.
\end{abstract}

Key words: Fabry disease, multiple myeloma, AL amyloidosis, enzyme replacement therapy, hemodialysis, monoclonal gammopathy

(Intern Med 56: 841-846, 2017)

(DOI: 10.2169/internalmedicine.56.7623)

\section{Introduction}

Fabry disease (FD) is an X-linked inherited lysosomal disorder that is characterized by a deficiency in the $\alpha$ galactosidase A enzyme, which results in the abnormal accumulation of glycosphingolipids, mainly globotriaosylceramide (GL-3) (1). The estimated prevalence of FD is 1 in 40,000-170,000 live births (2). However, recent newborn and high-risk group screening studies have suggested that the prevalence of non-classical FD is much higher than previously thought $(3,4)$.

The early symptoms of FD, which appear during childhood and adolescence, include angiokeratoma, anhydrosis, extremity pain, gastrointestinal symptoms, and proteinuria. Progressive renal failure, heart failure, and neurological dysfunction generally occur in adulthood. Notably, in patients with FD, a shortened life expectancy and morbidity are strongly associated with the degree of end-stage organ dysfunction.
Currently, two enzyme preparations, agalsidase alfa (Shire HGT, Boston, MA, USA) and agalsidase beta (Genzyme Corporation, Boston, MA, USA) are available for the treatment of FD. Hemizygous FD patients (men) usually develop renal insufficiency at roughly 40-50 years of age in the absence of enzyme replacement therapy (ERT). On the other hand, an initial clinical trial demonstrated that ERT could stabilize kidney function (5) and prevent the progression of cardiomyopathy (6). Furthermore, Pisani et al. showed that ERT was safe and effective, even for hemodialysis (HD) patients, and that it could improve the global quality of life and possibly ameliorate the progression of FD-related cardiomyopathy (7). Because of the increase in the life expectancy of FD patients due to ERT, it is likely that FD might accompany seemingly unrelated diseases. Accordingly, FD patients, who present with various clinical features and multi-organ dysfunction, tend to pose a diagnostic challenge for nephrologists, and this tendency is expected to increase in the future. We herein describe our recent encounter with a HD patient with FD who was receiving ERT and who suf-

${ }^{1}$ Division of Nephrology, Department of Medicine, Kurume University School of Medicine, Japan and ${ }^{2}$ Moriyama Clinic of Internal Medicine, Japan

Received for publication April 24, 2016; Accepted for publication August 3, 2016

Correspondence to Dr. Kensei Taguchi, taguchi_kensei@med.kurume-u.ac.jp 


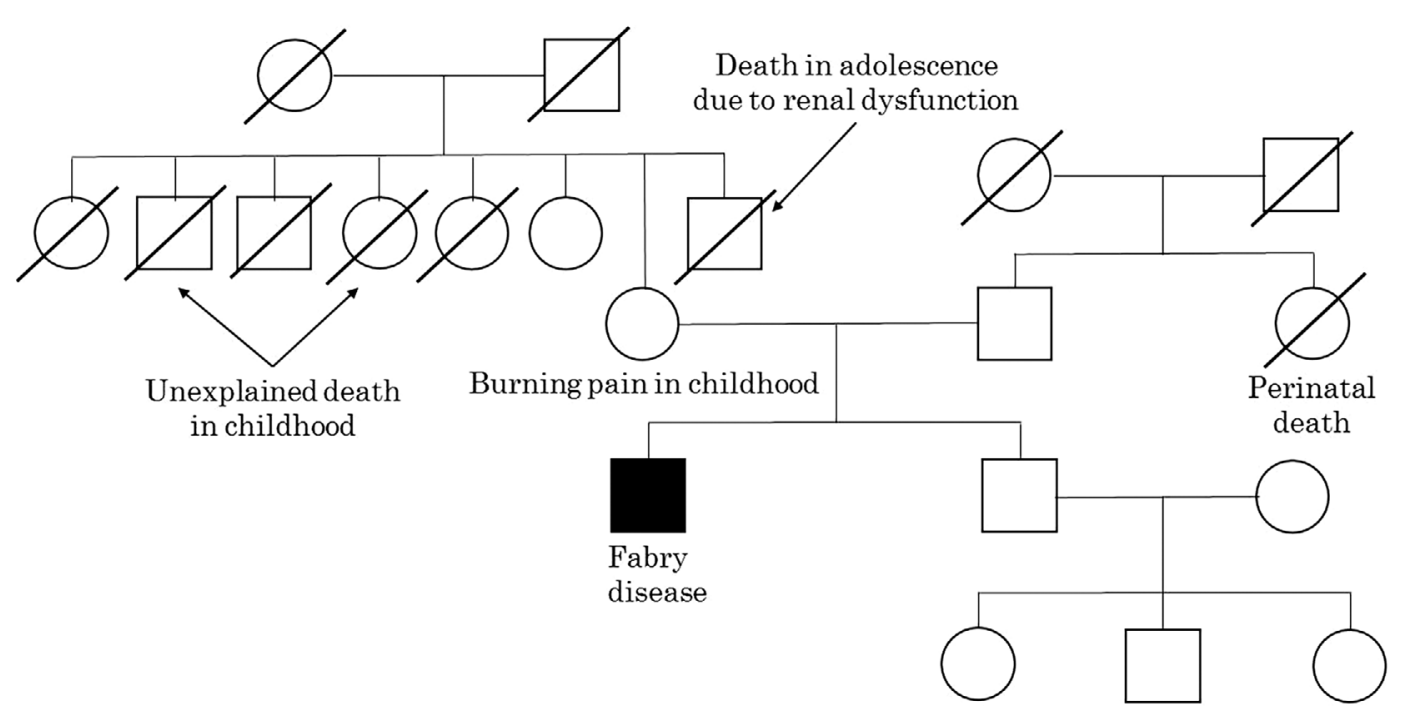

Figure 1. The family pedigree relevant to the present case. The patient is indicated by a black square.

fered from amyloid light-chain (AL) amyloidosis due to multiple myeloma (MM). Although few reports have described these coexisting conditions, we present our case along with a review of the relevant literature.

\section{Case Report}

The patient in the present case was a 50-year-old man who had received maintenance HD for 19 years. Although the patient had not undergone a genetic evaluation, a family history analysis revealed that his mother had suffered from burning pain in her extremities; in addition, some maternal siblings had died of unexplained causes during childhood (Fig. 1). The patient had initially presented with anhidrosis and burning upper extremity pain in childhood, at which time a physical examination revealed angiokeratoma around the lower abdomen and cornea verticillata (Fig. 2A). He was later diagnosed with FD via a muscle biopsy at 21 years of age. ERT (agalsidase beta, $60 \mathrm{mg} / \mathrm{month}$ ), which was initiated when he was 40 years of age, slightly attenuated the anhidrosis and burning extremity pain. However, he developed difficulty in speaking due to macroglossia (Fig. 2B), and reported epigastric discomfort and anorexia in June 2014. This patient received regular HD at our institution, which allowed us to monitor his condition between examinations. Unfortunately, treatment with metoclopramide failed to improve his symptoms, which gradually worsened during the few months before the next examination.

We performed an upper gastrointestinal endoscopy, which revealed multiple ulcers in the pyloric zone (Fig. 2B). Abdominal ultrasonography also detected gastric mucosal hypertrophy. Hematoxylin and Eosin staining identified amyloid deposits in the gastric mucosa and submucosa that contained amorphous, homogeneous, translucent, and acidophilic material. Direct fast scarlet staining revealed orange amyloid deposits. In addition, an immunohistochemical analysis revealed amyloid $\mathrm{P}$, but not amyloid A, positivity, which was compatible with AL amyloidosis (Fig. 3). No other clinical features, such as amyloidosis-related hepatomegaly, demyelinating polyneuropathy, orthostatic hypotension or carpal-tunnel syndrome, were observed.

A further analysis revealed that the peak serum GL-3 level, measured just before the monthly administration of agalsidase beta, was $6.0 \mu \mathrm{g} / \mathrm{mL}$, which is within the optimal range. Similarly, the patient's serum $\beta 2$-microglobulin concentration was $5.5 \mathrm{mg} / \mathrm{L}$, which is comparable to the level of a normal HD patient. Neither progressive anemia nor hypercalcemia was observed. Radiography was performed but detected no osteolytic changes or compression fractures. However, we identified abnormalities in the patient's high free light-chain (FLC) level and total $\kappa$ to $\lambda$ ratio $(\kappa / \lambda)$. The serum $\operatorname{IgG}, \operatorname{IgA}$, and IgM levels were suppressed at 476 , 129 , and $60 \mathrm{mg} / \mathrm{dL}$, respectively. These features, together with the detection of M-protein [Bence-Jones Protein (BJP) $\kappa$ type] via serum protein electrophoresis, led us to suspect MM. After bone marrow aspiration and biopsy revealed a $23 \%$ increase in plasma cells and abnormal binucleate plasma cells (Fig. 4), we diagnosed the patient with AL amyloidosis due to MM.

Electrocardiography showed increased QRS complex voltages and giant negative $\mathrm{T}$ waves, which are typical features of FD-related cardiomyopathy (Fig. 5A). A two-dimensional echocardiogram analysis revealed severe left ventricular hypertrophy (LVH) (Fig. 5B) and the preservation of the systolic function [ejection fraction (Teichholts MM): 70.8\%]. A Doppler evaluation revealed that the E/e' ratio was increased to 21.2 , and the patient's serum human atrial natriuretic peptide level was elevated to $88.7 \mathrm{pg} / \mathrm{mL}$, suggesting the existence of severe diastolic dysfunction, possibly due to FDrelated cardiomyopathy. However, the patient had not yet presented with severe congestive heart failure. In addition, no "sparkling or speckled appearance" was detected in the 

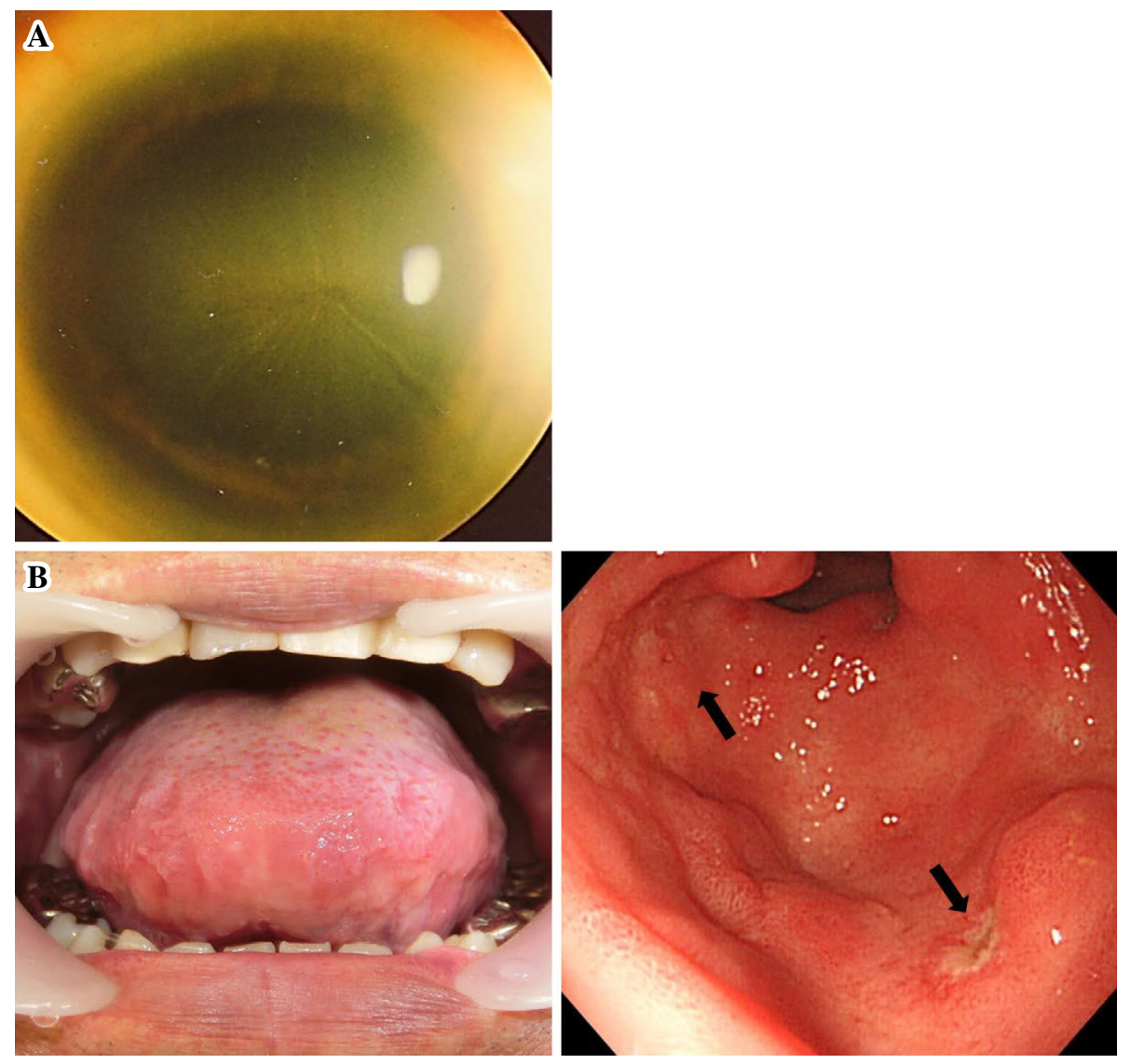

Figure 2. A: The slit-lamp appearance of the right cornea 31 years previously; cornea verticillata is visible. B: Left, macroglossia with indentations caused by teeth. Right, multiple ulcers in the pyloric zone (black arrows).

left ventricular wall; in other words, these examinations provided no evidence of cardiac amyloidosis.

Bortezomib and dexamethasone (BD) therapy was initiated. Bortezomib $\left(1.3 \mathrm{mg} / \mathrm{m}^{2}\right)$ was administered subcutaneously on days $1,8,15$, and 22 of a 5-week cycle (onceweekly administration), and dexamethasone (20 mg) was administered orally on the day of bortezomib treatment and the following day. After the third course of BD therapy, the patient's FLC concentration and $\kappa / \lambda$ ratio were decreased $[3,810.0$ to $75.0(\mathrm{mg} / \mathrm{L}), 94.78$ to 1.16 , respectively], and serum $\mathrm{M}$ protein was not detected.

After the fourteenth course of BD therapy, his gastrointestinal manifestations gradually improved, leading to an increased dietary intake. His macroglossia was also attenuated, thus eliminating his speaking difficulty. However, the patient remains under close observation with regard to lower extremity palsy, which is considered to be a side effect of bortezomib.

\section{Discussion}

FD, Gaucher disease, and Pompe disease are the most common lysosomal storage disorders. Among these conditions, Gaucher disease type I has a strong relationship with gammopathies such as $\mathrm{MM}$ and $\mathrm{AL}$ amyloidosis (8). Although the mechanism underlying this relationship has not been fully elucidated, ERT has been shown to ameliorate the increase in the serum concentrations of each immunoglobulin in patients with Gaucher disease (9). Indeed, Nair et al. demonstrated that in patients with Gaucher disease and a mouse model of Gaucher disease-associated gammopathy, clonal immunoglobulin reacts against lyso-glucosylceramide (LGL1); both levels were found to be markedly elevated in patients and mice. Furthermore, LGL1 reduction therapy ameliorated Gaucher disease-associated gammopathy in mice (10), suggesting that the accumulation of glycosphingolipid might be essential for the development of gammopathy. Although the type of glycosphingolipid accumulation that occurs in FD is different, the reaction of clonal immunoglobulin against GL-3 may also be associated with FDrelated gammopathy. In fact, a synchronous presentation of FD and monoclonal gammopathy, in which ERT stabilized serum M-protein levels without myeloma therapy, was previously reported (11). These observations indicate that the monoclonal immunoglobulin in FD patients might be reactive against GL-3; thus, its reduction by ERT could prevent monoclonal gammopathy. However, it has been shown that ERT failed to slow disease progression among FD patients who were older than 40 years of age, suggesting that ERT could not completely remove GL-3, which had already accumulated in the organ tissues (12). The patient in the present case started ERT at 40 years of age, thus the delayed initia- 

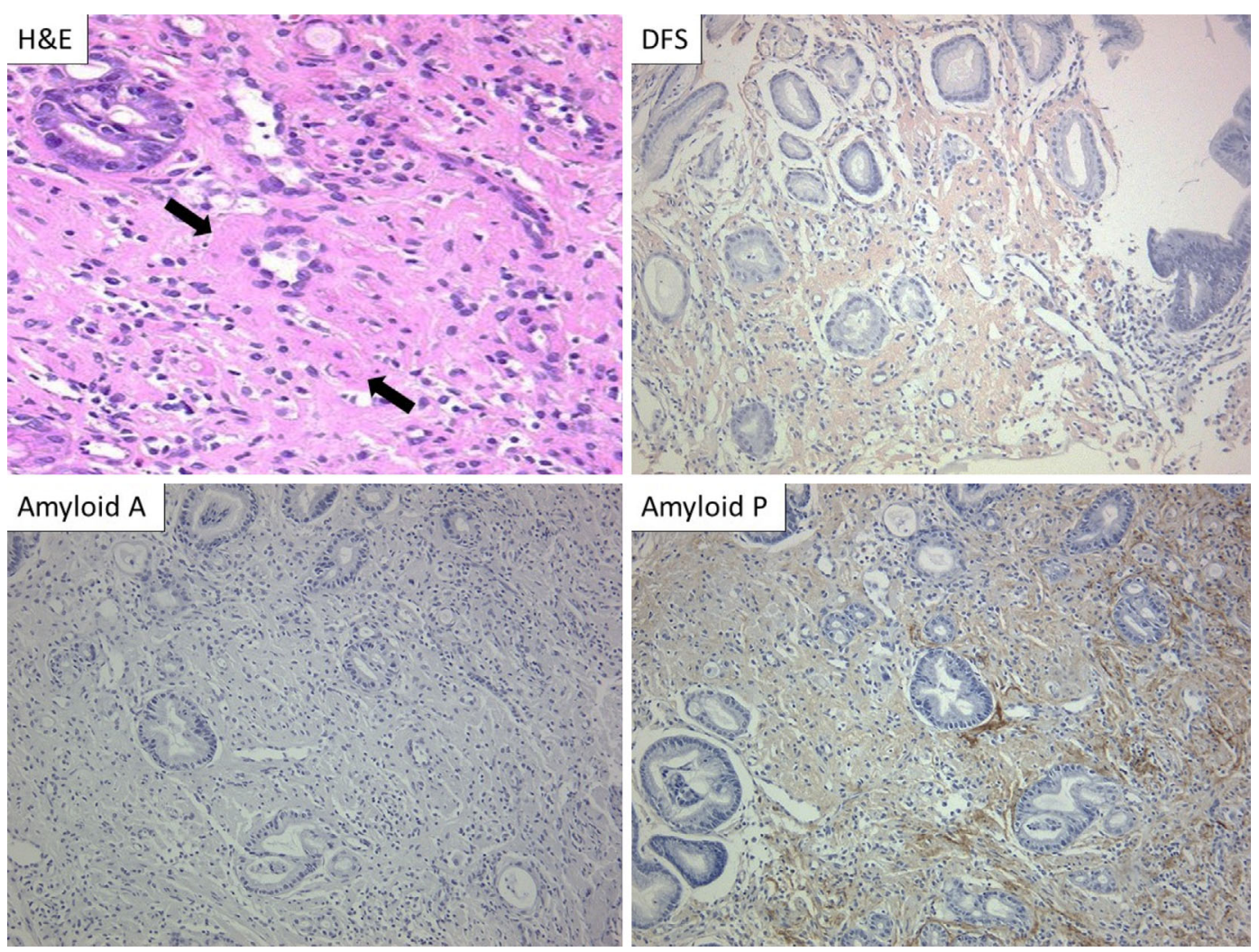

Figure 3. Representative images of the gastric mucosa in the patient. Magnification, $\times 200$. The amorphous homogeneous and acidophilic extracellular substance deposits that were visible in an Hematoxylin and Eosin staining specimen (upper left) were found to contain accumulated amyloid protein (black arrows). A DFS (upper right) confirmed the accumulation of amyloid in these deposits. Amyloid A staining was negative (lower left), whereas a amyloid P staining was positive (lower right), which confirmed a final diagnosis of amyloid light-chain amyloidosis. DFS: direct fast scarlet stain
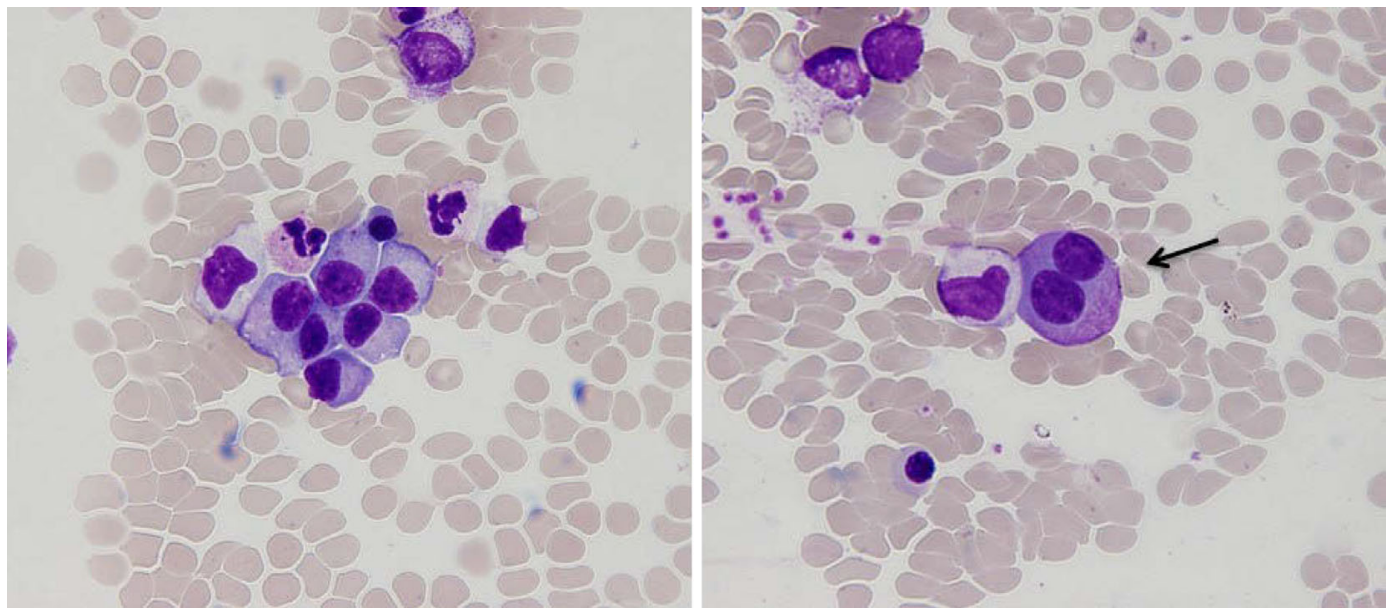

Figure 4. Bone marrow aspiration revealed numerous plasma cells, which can be recognized by the presence of eccentric nuclei and perinuclear halos. Abnormal binucleate plasma cells were observed (arrow; May-Grunwald Giemsa staining; magnification, $\times 600$ ).

tion of ERT might have caused the occurrence of MM in spite of longitudinal ERT.

The present case raised several valid concerns regarding the diagnoses of $\mathrm{MM}, \mathrm{AL}$ amyloidosis and FD requiring HD. First, HD normalized the patient's laboratory findings, thus hindering the diagnosis of MM. Specifically, a diagno- sis of symptomatic myeloma according to the International Multiple Myeloma Working Group criteria requires at least one of the following findings: an elevated calcium level $(>11.5 \mathrm{mg} / \mathrm{dL}$ ), renal insufficiency (serum creatinine $>2.0$ $\mathrm{mg} / \mathrm{dL}$ ), anemia (hemoglobin $<10 \mathrm{~g} / \mathrm{dL}$ or $2 \mathrm{~g} / \mathrm{dL}$ below normal), and bone disease (13). The biochemical parameters 

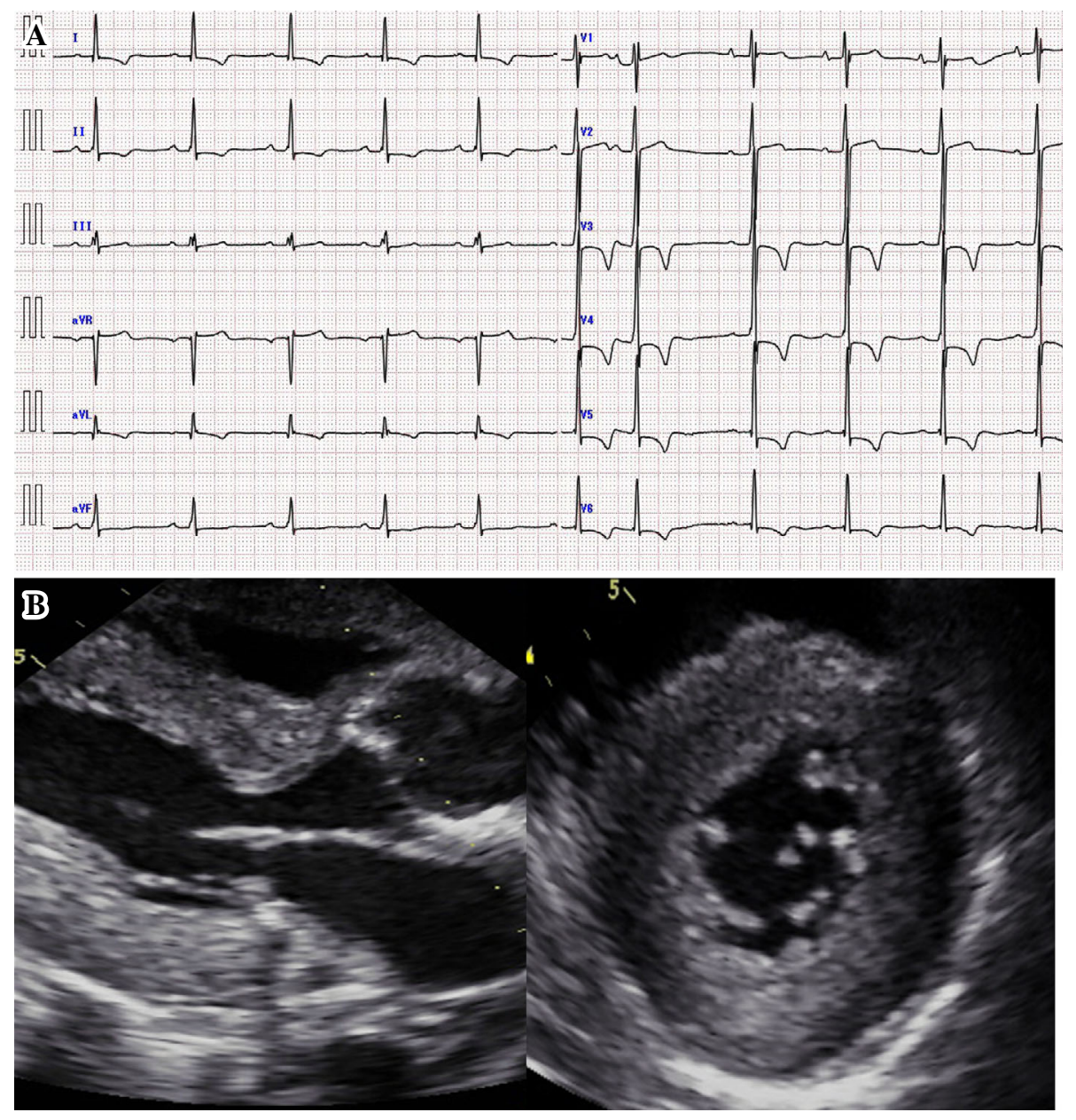

Figure 5. A: An electrocardiogram showed increased QRS complex voltages and a giant negative T wave in the precordial leads. B: A two-dimensional echocardiogram analysis revealed severe left ventricular hypertrophy, but no "sparkling or speckled appearance".

are generally normalized by $\mathrm{HD}$ and the administration of erythropoiesis-stimulating agents in subjects with HD. In comparison with these parameters, the measurement of FLC clearly provided us with an accurate diagnosis of MM in this patient. A growing body of evidence demonstrates that the serum FLC concentration and FLC $\kappa / \lambda$ ratio are valuable components in diagnosing $\mathrm{MM}$ in the general population. Notably, neither HD nor hemodiafiltration has been shown to affect the serum FLC concentration or $\kappa / \lambda$ ratio in patients with end-stage renal disease (14), indicating that these parameters are convenient, sensitive, and specific to MM, even in patients with HD.

Secondly, the similarity of the gastrointestinal manifestations of FD and AL amyloidosis might have contributed to diagnostic difficulty. Banikazemi et al. reported three FD patients with post-prandial abdominal pain, bloating and severe diarrhea. Agalsidase beta therapy markedly improved the gastrointestinal symptoms in all three patients after 6-7 months, and these significant improvements continued for more than 3 years of treatment (15). We should therefore consider the presence of newly identified concomitant diseases when gastrointestinal manifestations occur in FD patients, especially those who receive agalsidase beta therapy.
In conclusion, we presented the first case report describing the coexistence of FD and MM. Clinicians should therefore note that while the causal relationship between FD and MM has not been clarified, MM and consequent AL amyloidosis might be a cause of gastrointestinal manifestations in FD patients. This knowledge could permit the early diagnosis of such comorbidities and thereby prevent a poor prognosis in patients with FD.

The authors state that they have no Conflict of Interest (COI).

\section{References}

1. Desnick R. The Metabolic and Molecular Bases of Inherited Disease Vol. 3. 8th Ed. McGraw Hill, New York, 2001: 3733-3774.

2. Meikle PJ, Hopwood JJ, Clague AE, Carey WF. Prevalence of lysosomal storage disorders. JAMA 281: 249-254, 1999.

3. Mechtler TP, Stary S, Metz TF, et al. Neonatal screening for lysosomal storage disorders: feasibility and incidence from a nationwide study in Austria. Lancet 28; 379: 335-341, 2012.

4. Spada M, Pagliardini S, Yasuda M, et al. High incidence of lateronset Fabry disease revealed by newborn screening. Am J Hum Genet 79: 31-40, 2006.

5. Schiffmann R, Kopp JB, Austin HA 3rd, et al. Enzyme replace- 
ment therapy in Fabry disease: a randomized controlled trial. JAMA 285: 2743-2749, 2001.

6. Feriozzi S, Torras J, Cybulla M, et al. The effectiveness of longterm agalsidase alfa therapy in the treatment of Fabry nephropathy. Clin J Am Soc Nephrol 7: 60-69, 2012.

7. Pisani A, Spinelli L, Sabbatini M, et al. Enzyme replacement therapy in Fabry disease patients undergoing dialysis: effects on quality of life and organ involvement. Am J Kidney Dis 46: 120-127, 2005.

8. de Fost M, Out TA, de Wilde FA, et al. Immunoglobulin and free light chain abnormalities in Gaucher disease type I: data from an adult cohort of 63 patients and review of the literature. Ann Hematol 87: 439-449, 2008.

9. Brautbar A, Elstein D, Pines G, Abrahamov A, Zimran A. Effect of enzyme replacement therapy on gammopathies in Gaucher disease. Blood Cells Mol Dis 32: 214-217, 2004.

10. Nair S, Branagan AR, Liu J, Boddupalli CS, Mistry PK, Dhodapkar MV. Clonal immunoglobulin against lysolipids in the origin of myeloma. N Engl J Med 374: 555-561, 2016.

11. Pokuri VK, Xu B, Neppalli V, et al. Synchronous presentation of monoclonal gammopathy and Fabry nephropathy; diagnostic renal biopsy obviates initiation of myeloma therapy. Ann Hematol 94: 1067-1068, 2015.

12. Germain DP, Waldek S, Banikazemi M, et al. Sustained, long-term renal stabilization after 54 months of agalsidase $\beta$ therapy in patients with Fabry disease. J Am Soc Nephrol 18: 1547-1557, 2007.

13. International Myeloma Working Group. Criteria for the classification of monoclonal gammopathies, multiple myeloma and related disorders: a report of the International Myeloma Working Group. Br J Haematol 121: 749-757, 2003.

14. Cohen G, Rudnicki M, Schmaldienst S, Hörl WH. Effect of dialysis on serum/plasma levels of free immunoglobulin light chains in end-stage renal disease patients. Nephrol Dial Transplant 17: 879883, 2002.

15. Banikazemi M, Ullman T, Desnick RJ. Gastrointestinal manifestations of Fabry disease: clinical response to enzyme replacement therapy. Mol Genet Metab 85: 255-259, 2005.

The Internal Medicine is an Open Access article distributed under the Creative Commons Attribution-NonCommercial-NoDerivatives 4.0 International License. To view the details of this license, please visit (https://creativecommons.org/licenses/ by-nc-nd/4.0/).

(C) 2017 The Japanese Society of Internal Medicine http://www.naika.or.jp/imonline/index.html 